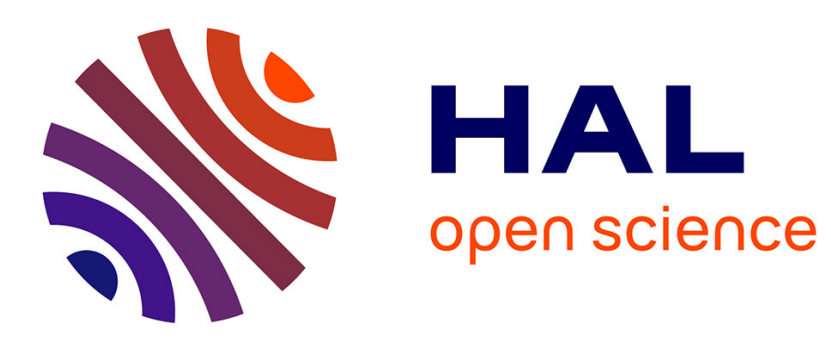

\title{
OBSERVATIONS DU MONOXYDE DE CARBONE DANS M31
}

F. Combes, P. Encrenaz, R. Lucas, L. Weliachew

\section{To cite this version:}

F. Combes, P. Encrenaz, R. Lucas, L. Weliachew. OBSERVATIONS DU MONOXYDE DE CARBONE DANS M31. Journal de Physique Colloques, 1978, 39 (C1), pp.C1-107-C1-108. 10.1051/jphyscol:1978117 . jpa-00217313

\section{HAL Id: jpa-00217313 https://hal.science/jpa-00217313}

Submitted on 1 Jan 1978

HAL is a multi-disciplinary open access archive for the deposit and dissemination of scientific research documents, whether they are published or not. The documents may come from teaching and research institutions in France or abroad, or from public or private research centers.
L'archive ouverte pluridisciplinaire HAL, est destinée au dépôt et à la diffusion de documents scientifiques de niveau recherche, publiés ou non, émanant des établissements d'enseignement et de recherche français ou étrangers, des laboratoires publics ou privés. 


\title{
OBSERVATIONS DU MONOXYDE DE CARBONE DANS MBI
}

\author{
F. COMBES, P. J. ENCRENAZ, R. LUCAS \\ Laboratoire de Physique, Ecole Normale Supérieure \\ et Département de Radioastronomie, Observatoire de Meudon, 92190 Meudon, France \\ et L. WELIACHEW \\ Département de Radioastronomie, Observatoire de Meudon, 92190 Meudon, France
}

\begin{abstract}
Résumé. - L'émission du monoxyde de carbone a été détectée dans les bras spiraux de la galaxie Andromède (M31). Sa distribution radiale le long du grand axe est partiellement obtenue et montre que l'émission de $\mathrm{CO}$ se trouve à l'intérieur des bras spiraux définis par l'hydrogène neutre.
\end{abstract}

\begin{abstract}
Emission from carbon monoxide has been detected in the spiral arms of the galaxy Andromeda (M31), and a partial radial distribution along the major axis is presented. This latter shows that CO emission is only found in the inside of the HI spiral arms.
\end{abstract}

Ce n'est que récemment que le monoxyde de carbone a été découvert dans les galaxies extérieures. Les premières recherches ont été effectuées en direction du noyau des galaxies, le noyau de notre galaxie étant une forte source d'émission $\mathrm{CO}$. Seules certaines galaxies, NGC253, M82 et M51 par exemple, présentent une émission vers le centre [1] : en particulier aucune émission n'a été détectée au centre de M31 où la profonde dépression en hydrogène neutre [2] et l'absence de régions HII [3] pouvaient suggérer que l'hydrogène était sous forme moléculaire.

Dans notre galaxie, le monoxyde de carbone est aussi détecté dans une région annulaire entre $4 \mathrm{kpc}$ et $8 \mathrm{kpc}$ du centre ; associé aux régions les plus denses, il pourrait être un bon traceur de la structure spirale. Dans les galaxies extérieures, l'émission doit être très affaiblie par le facteur de dilution dans le lobe des antennes actuelles (typiquement 1 minute d'arc) : la source attendue est inhomogène, composée de nombreux nuages moléculaires de faible dimension (de l'ordre de $10 \mathrm{pc}$ ). De plus la région de ces nuages, si elle est restreinte à l'intérieur des bras spiraux, est peu étendue, d'où l'importance d'observer des galaxies proches pour éviter une plus grande dilution.

Andromède est la galaxie spirale la plus proche de la nôtre. La première détection de CO dans M31 a été effectuée dans les bras spiraux, par F. Combes, P. J. Encrenaz, R. Lucas, L. Weliachew [4] en novembre 1976. Ces observations, complétées en juin 1977 ont été effectuées avec l'antenne de $11 \mathrm{~m}$ de l'Observatoire de Kitt Peak, N. R. A. O. $\left(^{*}\right)$; le lobe à mipuissance est de $64^{\prime \prime}$ à la fréquence de $115 \mathrm{GHz}$, la résolution en vitesses était de $2,6 \mathrm{~km} / \mathrm{s}$ et $1,3 \mathrm{~km} / \mathrm{s}$ correspondant aux deux filtres utilisés. La précision de pointage était de 7 ".

Les résultats sont rassemblés dans la distribution radiale de la figure 1 , superposés sur la distribution d'hydrogène neutre de J. Guibert [5]. Il apparaît nettement sur cette figure que l'émission de $\mathrm{CO}$ est concentrée à l'intérieur des bras délimités par l'hydrogène atomique, schéma attendu par la théorie des ondes de densité : au passage à travers un bras spiral la matière est comprimée, se condense en partie et des nuages moléculaires se forment, précisément à l'intérieur des bras; après cette compression, souvent accompagnée d'une onde de choc et se produisant sur une très petite échelle, suit l'expansion du gaz neutre, région plus étendue qui contribue aux maxima de la distribution HI (Fig. 1 par exemple).

Les spectres $\mathrm{CO}$ obtenus ont des températures d'antennes allant de $0,1 \mathrm{~K}$ à $0,3 \mathrm{~K}$, températures Rayleigh-Jeans corrigées de l'atténuation atmosphérique et des pertes d'antenne; l'émission est plus forte dans la partie sud de la galaxie.

(*) Le National Radio Astronomy Observatory est opéré par Associated Universities Inc., sous contrat avec la National Science Foundation. 


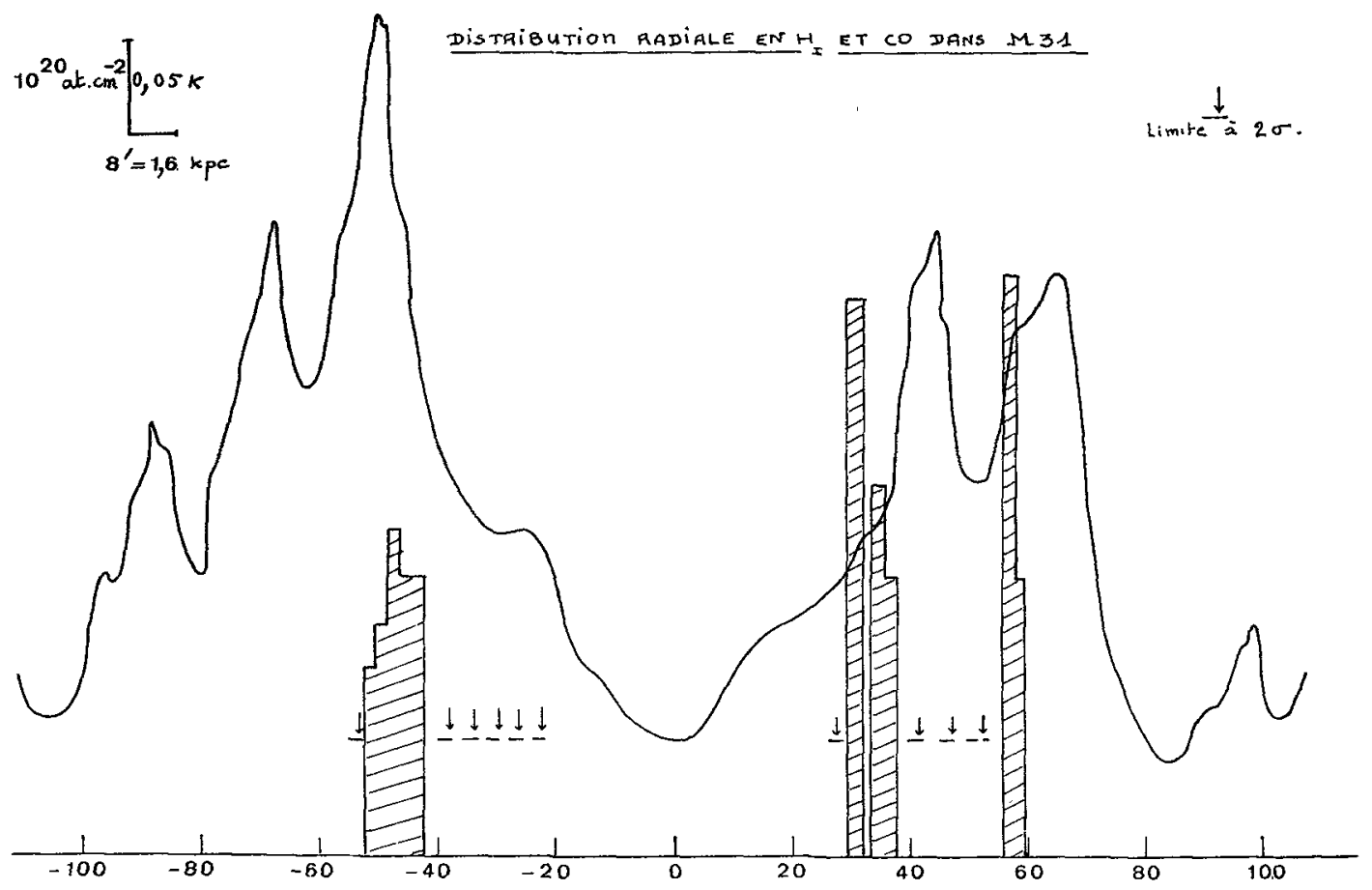

FIG. 1. - Distribution radiale en CO et hydrogène atomique dans M31. La distribution d'hydrogène est la densité de surface le long du grand axe, tirée des observations de J. Guibert [5]; les histogrammes hachurés représentent les températures d'antenne obtenues en $\mathrm{CO}$, températures Rayleigh-Jeans corrigées de l'atténuation atmosphérique et des pertes đ'antenne.

Les profils obtenus sont assez étroits, de 10 à $25 \mathrm{~km} / \mathrm{s}$, surtout en comparaison avec les profils obtenus vers le centre des galaxies. La largeur de ces profils correspond au gradient de vitesse en hydrogène neutre. $\mathrm{La}$ vitesse du centre de gravité des profils $\mathrm{CO}$ est très proche de celle de l'hydrogène; toutefois une légère différence systématique a été détectée, suggérant une plus grande vitesse de rotation pour le monoxyde de carbone que pour l'hydrogène, au nord comme au sud. Il est nécessaire de disposer d'observations $\mathrm{HI}$ à plus haute résolution spatiale et en vitesse pour confirmer la réalité de tels effets.

\section{Bibliographie}

[1] Rickard, L. J., Palmer, P., Morris, M., Zuckerman, B., TURNER, B. E., Astrophys. J. 199 (1975) L 75.

[2] GURBert, J., Astron. Astrophys. 29 (1973) 335.

[3] Monnet, G., Astron. Astrophys. 12 (1971) 379.
[4] Combes, F., Encrenaz, P. J., Lucas, R., Weliachew, L., Astron. Astrophys. 55 (1977) 311.

[5] Gurbert, J., Colloque de Bures, Editions C.N.R.S. (1975) 266. 